\title{
An Extragradient Approximation Method for Equilibrium Problems and Fixed Point Problems of a Countable Family of Nonexpansive Mappings
}

\author{
Rabian Wangkeeree \\ Department of Mathematics, Faculty of Science, Naresuan University, Phitsanulok 65000, Thailand \\ Correspondence should be addressed to Rabian Wangkeeree, rabianw@nu.ac.th \\ Received 28 February 2008; Accepted 13 July 2008 \\ Recommended by Huang Nanjing
}

\begin{abstract}
We introduce a new iterative scheme for finding the common element of the set of common fixed points of nonexpansive mappings, the set of solutions of an equilibrium problem, and the set of solutions of the variational inequality. We show that the sequence converges strongly to a common element of the above three sets under some parameters controlling conditions. Moreover, we apply our result to the problem of finding a common fixed point of a countable family of nonexpansive mappings, and the problem of finding a zero of a monotone operator. This main theorem extends a recent result of Yao et al. (2007) and many others.
\end{abstract}

Copyright (c) 2008 Rabian Wangkeeree. This is an open access article distributed under the Creative Commons Attribution License, which permits unrestricted use, distribution, and reproduction in any medium, provided the original work is properly cited.

\section{Introduction}

Let $H$ be a real Hilbert space with inner product $\langle\cdot, \cdot\rangle$ and norm $\|\cdot\|$, and let $C$ be a closed convex subset of $H$. Let $F$ be a bifunction of $C \times C$ into $\mathbb{R}$, where $\mathbb{R}$ is the set of real numbers. The equilibrium problem for $\phi: C \times C \rightarrow \mathbb{R}$ is to find $x \in C$ such that

$$
\phi(x, y) \geq 0 \quad \forall y \in C .
$$

The set of solutions of (1.1) is denoted by $\operatorname{EP}(\phi)$. Given a mapping $T: C \rightarrow H$, let $\phi(x, y)=$ $\langle T x, y-x\rangle$ for all $x, y \in C$. Then $z \in \operatorname{EP}(\phi)$ if and only if $\langle T z, y-z\rangle \geq 0$ for all $y \in C$, that is, $z$ is a solution of the variational inequality. Numerous problems in physics, optimization, and economics reduce to find a solution of (1.1). In 1997, Flåm and Antipin [1] introduced an iterative scheme of finding the best approximation to initial data when $\operatorname{EP}(\phi)$ is nonempty and proved a strong convergence theorem.

Let $A: C \rightarrow H$ be a mapping. The classical variational inequality, denoted by $\operatorname{VI}(A, C)$, is to find $x^{*} \in C$ such that

$$
\left\langle A x^{*}, v-x^{*}\right\rangle \geq 0
$$


for all $v \in C$. The variational inequality has been extensively studied in the literature. See, for example, $[2,3]$ and the references therein. A mapping $A$ of $C$ into $H$ is called $\alpha$-inversestrongly monotone $[4,5]$ if there exists a positive real number $\alpha$ such that

$$
\langle A u-A v, u-v\rangle \geq \alpha\|A u-A v\|^{2}
$$

for all $u, v \in C$. It is obvious that any $\alpha$-inverse-strongly monotone mapping $A$ is monotone and Lipschitz continuous. A mapping $S$ of $C$ into itself is called nonexpansive if

$$
\|S u-S v\| \leq\|u-v\|
$$

for all $u, v \in C$. We denote by $F(S)$ the set of fixed points of $S$. For finding an element of $F(S) \cap \operatorname{VI}(A, C)$, under the assumption that a set $C \subseteq H$ is nonempty, closed, and convex, a mapping $S: C \rightarrow C$ is nonexpansive and a mapping $A: C \rightarrow H$ is $\alpha$-inverse-strongly monotone, Takahashi and Toyoda [6] introduced the following iterative scheme:

$$
x_{n+1}=\alpha_{n} x_{n}+\left(1-\alpha_{n}\right) S P_{C}\left(x_{n}-\lambda_{n} A x_{n}\right)
$$

for every $n=0,1,2, \ldots$, where $x_{0}=x \in C,\left\{\alpha_{n}\right\}$ is a sequence in $(0,1)$, and $\left\{\lambda_{n}\right\}$ is a sequence in $(0,2 \alpha)$. They proved that if $F(S) \cap \operatorname{VI}(A, C) \neq \varnothing$, then the sequence $\left\{x_{n}\right\}$ generated by (1.5) converges weakly to some $z \in F(S) \cap \mathrm{VI}(A, C)$. Recently, motivated by the idea of Korpelevič's extragradient method [7], Nadezhkina and Takahashi [8] introduced an iterative scheme for finding an element of $F(S) \cap \operatorname{VI}(A, C)$ and the weak convergence theorem is presented. Moreover, Zeng and Yao [9] proposed some new iterative schemes for finding elements in $F(S) \cap \operatorname{VI}(A, C)$ and obtained the weak convergence theorem for such schemes. Very recently, Yao et al. [10] introduced the following iterative scheme for finding an element of $F(S) \cap \operatorname{VI}(A, C)$ under some mild conditions. Let $C$ be a closed convex subset of a real Hilbert space $H, A: C \rightarrow H$ a monotone, $L$-Lipschitz continuous mapping, and $S$ a nonexpansive mapping of $C$ into itself such that $F(S) \cap \operatorname{VI}(A, C) \neq \varnothing$. Suppose that $x_{1}=u \in C$ and $\left\{x_{n}\right\},\left\{y_{n}\right\}$ are given by

$$
\begin{gathered}
y_{n}=P_{C}\left(x_{n}-\lambda_{n} A x_{n}\right), \\
x_{n+1}=\alpha_{n} u+\beta_{n} x_{n}+\gamma_{n} S P_{C}\left(x_{n}-\lambda_{n} A y_{n}\right) \quad \forall n \in \mathbb{N},
\end{gathered}
$$

where $\left\{\alpha_{n}\right\},\left\{\beta_{n}\right\},\left\{\gamma_{n}\right\} \subseteq[0,1]$ and $\left\{\lambda_{n}\right\} \subseteq(0,1)$ satisfy some parameters controlling conditions. They proved that the sequence $\left\{x_{n}\right\}$ defined by (1.6) converges strongly to a common element of $F(S) \cap \operatorname{VI}(A, C)$.

On the other hand, S. Takahashi and W. Takahashi [11] introduced an iterative scheme by the viscosity approximation method for finding a common element of the set of solution (1.1) and the set of fixed points of a nonexpansive mapping in a real Hilbert space. Let $S$ : $C \rightarrow C$ be a nonexpansive mapping. Starting with arbitrary initial $x_{1} \in C$, define sequences $\left\{x_{n}\right\}$ and $\left\{u_{n}\right\}$ recursively by

$$
\begin{gathered}
\phi\left(u_{n}, y\right)+\frac{1}{r_{n}}\left\langle y-u_{n}, u_{n}-x_{n}\right\rangle \geq 0 \quad \forall y \in C, \\
x_{n+1}=\alpha_{n} f\left(x_{n}\right)+\left(1-\alpha_{n}\right) S u_{n} \quad \forall n \in \mathbb{N} .
\end{gathered}
$$

They proved that under certain appropriate conditions imposed on $\left\{\alpha_{n}\right\}$ and $\left\{r_{n}\right\}$, the sequences $\left\{x_{n}\right\}$ and $\left\{u_{n}\right\}$ converge strongly to $z \in F(S) \cap \operatorname{EP}(\phi)$, where $z=P_{F(S) \cap E P(\phi)} f(z)$. 
Moreover, Aoyama et al. [12] introduced an iterative scheme for finding a common fixed point of a countable family of nonexpansive mappings in Banach spaces and obtained the strong convergence theorem for such scheme.

In this paper, motivated by Yao et al. [10], S. Takahashi and W. Takahashi [11] and Aoyama et al. [12], we introduce a new extragradient method (4.2) which is mixed the iterative schemes considered in [10-12] for finding a common element of the set of common fixed points of nonexpansive mappings, the set of solutions of an equilibrium problem, and the solution set of the classical variational inequality problem for a monotone $L$-Lipschitz continuous mapping in a real Hilbert space. Then, the strong convergence theorem is proved under some parameters controlling conditions. Further, we apply our result to the problem of finding a common fixed point of a countable family of nonexpansive mappings, and the problem of finding a zero of a monotone operator. The results obtained in this paper improve and extend the recent ones announced by Yao et al. results [10] and many others.

\section{Preliminaries}

Let $H$ be a real Hilbert space with norm $\|\cdot\|$ and inner product $\langle\cdot, \cdot \cdot\rangle$ and let $C$ be a closed convex subset of $H$. For every point $x \in H$, there exists a unique nearest point in $C$, denoted by $P_{C} x$, such that

$$
\left\|x-P_{C} x\right\| \leq\|x-y\| \quad \forall y \in C .
$$

$P_{C}$ is called the metric projection of $H$ onto $C$. It is well known that $P_{C}$ is a nonexpansive mapping of $H$ onto $C$ and satisfies

$$
\left\langle x-y, P_{C} x-P_{C} y\right\rangle \geq\left\|P_{C} x-P_{C} y\right\|^{2}
$$

for every $x, y \in H$. Moreover, $P_{C} x$ is characterized by the following properties: $P_{C} x \in C$ and

$$
\begin{gathered}
\left\langle x-P_{C} x, y-P_{C} x\right\rangle \leq 0, \\
\|x-y\|^{2} \geq\left\|x-P_{C} x\right\|^{2}+\left\|y-P_{C} x\right\|^{2}
\end{gathered}
$$

for all $x \in H, y \in C$. For more details, see [13]. It is easy to see that the following is true:

$$
u \in \mathrm{VI}(A, C) \Longleftrightarrow u=P_{C}(u-\lambda A u), \quad \lambda>0 .
$$

A set-valued mapping $T: H \rightarrow 2^{H}$ is called monotone if for all $x, y \in H, f \in T x$, and $g \in T y$ imply $\langle x-y, f-g\rangle \geq 0$. A monotone mapping $T: H \rightarrow 2^{H}$ is maximal if the graph of $G(T)$ of $T$ is not properly contained in the graph of any other monotone mapping. It is known that a monotone mapping $T$ is maximal if and only if for $(x, f) \in H \times H,\langle x-y, f-g\rangle \geq 0$ for every $(y, g) \in G(T)$ implies $f \in T x$. Let $B$ be a monotone map of $C$ into $H, L$-Lipschitz continuous mapping and let $N_{C} v$ be the normal cone to $C$ at $v \in C$, that is, $N_{C} v=\{w \in H$ : $\langle u-v, w\rangle \geq 0$ for all $u \in C\}$. Define

$$
T v= \begin{cases}B v+N_{C} v, & v \in C ; \\ \varnothing, & v \notin C .\end{cases}
$$

Then $T$ is the maximal monotone and $0 \in T v$ if and only if $v \in \operatorname{VI}(C, B)$; see [14]. 
The following lemmas will be useful for proving the convergence result of this paper.

Lemma 2.1 (see $[15])$. Let $(E,\langle\cdot, \cdot\rangle)$ be an inner product space. Then for all $x, y, z \in E$ and $\alpha, \beta, \gamma \in$ $[0,1]$ with $\alpha+\beta+\gamma=1$,one has

$$
\|\alpha x+\beta y+\gamma z\|^{2}=\alpha\|x\|^{2}+\beta\|y\|^{2}+\gamma\|z\|^{2}-\alpha \beta\|x-y\|^{2}-\alpha \gamma\|x-z\|^{2}-\beta \gamma\|y-z\|^{2} .
$$

Lemma 2.2 (see [16]). Let $\left\{x_{n}\right\}$ and $\left\{z_{n}\right\}$ be bounded sequences in a Banach space $E$ and let $\left\{\beta_{n}\right\}$ be a sequence in $[0,1]$ with $0<\liminf _{n \rightarrow \infty} \beta_{n} \leq \lim \sup _{n \rightarrow \infty} \beta_{n}<1$. Suppose $x_{n+1}=\left(1-\beta_{n}\right) z_{n}+\beta_{n} x_{n}$ for all integers $n \geq 1$ and $\lim \sup _{n \rightarrow \infty}\left(\left\|z_{n+1}-z_{n}\right\|-\left\|x_{n+1}-x_{n}\right\|\right) \leq 0$. Then, $\lim _{n \rightarrow \infty}\left\|z_{n}-x_{n}\right\|=0$.

Lemma 2.3 (see [17]). Assume $\left\{a_{n}\right\}$ is a sequence of nonnegative real numbers such that

$$
a_{n+1} \leq\left(1-\alpha_{n}\right) a_{n}+\delta_{n}, \quad n \geq 1
$$

where $\left\{\alpha_{n}\right\}$ is a sequence in $(0,1)$ and $\left\{\delta_{n}\right\}$ is a sequence in $\mathbb{R}$ suchthat

(i) $\sum_{n=1}^{\infty} \alpha_{n}=\infty$ and

(ii) $\lim \sup _{n \rightarrow \infty}\left(\delta_{n} / \alpha_{n}\right) \leq 0$ or $\sum_{n=1}^{\infty}\left|\delta_{n}\right|<\infty$.

Then $\lim _{n \rightarrow \infty} a_{n}=0$.

Lemma 2.4 (see [12, Lemma 3.2]). Let $C$ be a nonempty closed subset of a Banach space and let $\left\{S_{n}\right\}$ be a sequence of mappings of $C$ into itself. Suppose that $\sum_{n=1}^{\infty} \sup \left\{\left\|S_{n+1} z-S_{n} z\right\|: z \in C\right\}<\infty$. Then, for each $y \in C,\left\{S_{n} y\right\}$ converges strongly to some point of $C$. Moreover, let $S$ be a mapping of $C$ into itself defined by

$$
S y=\lim _{n \rightarrow \infty} S_{n} y \quad \forall y \in C .
$$

Then $\lim _{n \rightarrow \infty} \sup \left\{\left\|S z-S_{n} z\right\|: z \in C\right\}=0$.

For solving the equilibrium problem for a bifunction $\phi: C \times C \rightarrow \mathbb{R}$, let us assume that $\phi$ satisfies the following conditions:

(A1) $\phi(x, x)=0$ for all $x \in C$;

(A2) $\phi$ is monotone, that is, $\phi(x, y)+\phi(y, x) \leq 0$ for all $x, y \in C$;

(A3) for each $x, y, z \in C, \lim _{t \rightarrow 0} \phi(t z+(1-t) x, y) \leq \phi(x, y)$;

(A4) for each $x \in C, y \mapsto \phi(x, y)$ is convex and lower semicontinuous.

The following lemma appears implicitly in [18].

Lemma 2.5 (see [18]). Let $C$ be a nonempty closed convex subset of $H$ and let $\phi$ be a bifunction of $C \times C$ into $\mathbb{R}$ satisfying $(A 1)-(A 4)$. Let $r>0$ and $x \in H$. Then, there exists $z \in C$ such that

$$
\phi(z, y)+\frac{1}{r}\langle y-z, z-x\rangle \geq 0 \quad \forall y \in C
$$

The following lemma was also given in [1]. 
Lemma 2.6 (see [1]). Assume that $\phi: C \times C \rightarrow \mathbb{R}$ satisfies (A1)-(A4). For $r>0$ and $x \in H$, define a mapping $T_{r}: H \rightarrow C$ as follows:

$$
T_{r}(x)=\left\{z \in C: \phi(z, y)+\frac{1}{r}\langle y-z, z-x\rangle \geq 0 \forall y \in C\right\}
$$

for all $z \in H$. Then, the following hold:

(i) $T_{r}$ is single-valued;

(ii) $T_{r}$ is firmly nonexpansive, that is, for any $x, y \in H,\left\|T_{r} x-T_{r} y\right\|^{2} \leq\left\langle T_{r} x-T_{r} y, x-y\right\rangle$;

(iii) $F\left(T_{r}\right)=\mathrm{EP}(\phi)$;

(iv) $\mathrm{EP}(\phi)$ is closed and convex.

\section{Main results}

In this section, we prove a strong convergence theorem.

Theorem 3.1. Let $C$ be a closed convex subset of a real Hilbert space $H$. Let $\phi$ be a bifunction from $C \times$ $C$ to $\mathbb{R}$ satisfying $(A 1)-(A 4), A: C \rightarrow H$ a monotone L-Lipschitz continuous mapping and let $\left\{S_{n}\right\}$ be a sequence of nonexpansive mappings of $C$ into itself such that $\cap_{n=1}^{\infty} F\left(S_{n}\right) \cap \operatorname{VI}(A, C) \cap \mathrm{EP}(\phi) \neq \varnothing$. Let the sequences $\left\{x_{n}\right\},\left\{u_{n}\right\}$, and $\left\{y_{n}\right\}$ be generated by

$$
\begin{gathered}
x_{1}=x \in C \text { chosen arbitrarily, } \\
\phi\left(u_{n}, y\right)+\frac{1}{r_{n}}\left\langle y-u_{n}, u_{n}-x_{n}\right\rangle \geq 0 \quad \forall y \in C, \\
y_{n}=P_{C}\left(u_{n}-\lambda_{n} A u_{n}\right), \\
x_{n+1}=\alpha_{n} f\left(x_{n}\right)+\beta_{n} x_{n}+\gamma_{n} S_{n} P_{C}\left(u_{n}-\lambda_{n} A y_{n}\right) \quad \forall n \geq 1,
\end{gathered}
$$

where $\left\{\alpha_{n}\right\},\left\{\beta_{n}\right\},\left\{\gamma_{n}\right\} \subseteq[0,1],\left\{\lambda_{n}\right\} \subseteq(0,1)$, and $\left\{r_{n}\right\} \subseteq(0, \infty)$ satisfy the following conditions:

(C1) $\alpha_{n}+\beta_{n}+\gamma_{n}=1$,

(C2) $\lim _{n \rightarrow \infty} \alpha_{n}=0, \sum_{n=1}^{\infty} \alpha_{n}=\infty$,

(C3) $0<\liminf _{n \rightarrow \infty} \beta_{n} \leq \limsup _{n \rightarrow \infty} \beta_{n}<1$,

(C4) $\lim _{n \rightarrow \infty} \lambda_{n}=0$,

(C5) $\liminf _{n \rightarrow \infty} r_{n}>0, \sum_{n=1}^{\infty}\left|r_{n+1}-r_{n}\right|<\infty$.

Suppose that $\sum_{n=1}^{\infty} \sup \left\{\left\|S_{n+1} z-S_{n} z\right\|: z \in B\right\}<\infty$ for any bounded subset B of $C$. Let $S$ be a mapping of $C$ into itself defined by $S y=\lim _{n \rightarrow \infty} S_{n} y$ for all $y \in C$ and suppose that $F(S)=\cap_{n=1}^{\infty} F\left(S_{n}\right)$. Then the sequences $\left\{x_{n}\right\},\left\{u_{n}\right\}$, and $\left\{y_{n}\right\}$ converge strongly to the same point $q \in \cap_{n=1}^{\infty} F\left(S_{n}\right) \cap \operatorname{VI}(A, C) \cap \operatorname{EP}(\phi)$, where $q=P_{\cap_{n=1}^{\infty} F\left(S_{n}\right) \cap V I(A, C) \cap \operatorname{EP}(\phi)} f(q)$.

Proof. Let $Q=P_{\cap_{n=1}^{\infty}} F\left(S_{n}\right) \cap \mathrm{VI}(A, C) \cap \mathrm{EP}(\phi)$. Since $f$ is a contraction with $\alpha \in[0,1)$, we obtain

$$
\|Q f(x)-Q f(y)\| \leq\|f(x)-f(y)\| \leq \alpha\|x-y\| \quad \forall x, y \in C .
$$

Therefore, $Q f$ is a contraction of $C$ into itself, which implies that there exists a unique element $q \in C$ such that $q=Q f(q)$. Then we divide the proof into several steps. 
Step $1\left(\left\{x_{n}\right\}\right.$ is bounded). Indeed, put $t_{n}=P_{C}\left(u_{n}-\lambda_{n} A y_{n}\right)$ for all $n \geq 1$. Let $x^{*} \in \cap_{n=1}^{\infty} F\left(S_{n}\right) \cap$ $\operatorname{VI}(A, C) \cap \mathrm{EP}(\phi)$. From (2.5) we have $x^{*}=P_{C}\left(x^{*}-\lambda_{n} A x^{*}\right)$. Also it follows from (2.4) that

$$
\begin{aligned}
\left\|t_{n}-x^{*}\right\|^{2} \leq & \left\|u_{n}-\lambda_{n} A y_{n}-x^{*}\right\|^{2}-\left\|u_{n}-\lambda_{n} A y_{n}-t_{n}\right\|^{2} \\
= & \left\|u_{n}-x^{*}\right\|^{2}-2 \lambda_{n}\left\langle A y_{n}, u_{n}-x^{*}\right\rangle+\lambda_{n}^{2}\left\|A y_{n}\right\|^{2}-\left\|u_{n}-t_{n}\right\|^{2} \\
& +2 \lambda_{n}\left\langle A y_{n}, u_{n}-t_{n}\right\rangle-\lambda_{n}^{2}\left\|A y_{n}\right\|^{2} \\
= & \left\|u_{n}-x^{*}\right\|^{2}+2 \lambda_{n}\left\langle A y_{n}, x^{*}-t_{n}\right\rangle-\left\|u_{n}-t_{n}\right\|^{2} \\
= & \left\|u_{n}-x^{*}\right\|^{2}-\left\|u_{n}-t_{n}\right\|^{2}+2 \lambda_{n}\left\langle A y_{n}-A x^{*}, x^{*}-y_{n}\right\rangle \\
& +2 \lambda_{n}\left\langle A x^{*}, x^{*}-y_{n}\right\rangle+2 \lambda_{n}\left\langle A y_{n}, y_{n}-t_{n}\right\rangle .
\end{aligned}
$$

Since $A$ is monotone and $x^{*}$ is a solution of the variational inequality problem $\operatorname{VI}(A, C)$, we have

$$
\left\langle A y_{n}-A x^{*}, x^{*}-y_{n}\right\rangle \leq 0, \quad\left\langle A x^{*}, x^{*}-y_{n}\right\rangle \leq 0 .
$$

This together with (3.3) implies that

$$
\begin{aligned}
\left\|t_{n}-x^{*}\right\|^{2} & \leq\left\|u_{n}-x^{*}\right\|^{2}-\left\|u_{n}-t_{n}\right\|^{2}+2 \lambda_{n}\left\langle A y_{n}, y_{n}-t_{n}\right\rangle \\
& =\left\|u_{n}-x^{*}\right\|^{2}-\left\|\left(u_{n}-y_{n}\right)+\left(y_{n}-t_{n}\right)\right\|^{2}+2 \lambda_{n}\left\langle A y_{n}, y_{n}-t_{n}\right\rangle \\
& =\left\|u_{n}-x^{*}\right\|^{2}-\left\|u_{n}-y_{n}\right\|^{2}-2\left\langle u_{n}-y_{n}, y_{n}-t_{n}\right\rangle-\left\|y_{n}-t_{n}\right\|^{2}+2 \lambda_{n}\left\langle A y_{n}, y_{n}-t_{n}\right\rangle \\
& =\left\|u_{n}-x^{*}\right\|^{2}-\left\|u_{n}-y_{n}\right\|^{2}-\left\|y_{n}-t_{n}\right\|^{2}+2\left\langle u_{n}-\lambda_{n} A y_{n}-y_{n}, t_{n}-y_{n}\right\rangle .
\end{aligned}
$$

From (2.3), we have

$$
\left\langle u_{n}-\lambda_{n} A u_{n}-y_{n}, t_{n}-y_{n}\right\rangle \leq 0,
$$

so that

$$
\begin{aligned}
\left\langle u_{n}-\lambda_{n} A y_{n}-y_{n}, t_{n}-y_{n}\right\rangle & =\left\langle u_{n}-\lambda_{n} A u_{n}-y_{n}, t_{n}-y_{n}\right\rangle+\left\langle\lambda_{n} A u_{n}-\lambda_{n} A y_{n}, t_{n}-y_{n}\right\rangle \\
& \leq\left\langle\lambda_{n} A u_{n}-\lambda_{n} A y_{n}, t_{n}-y_{n}\right\rangle \\
& \leq \lambda_{n}\left\|A u_{n}-A y_{n}\right\|\left\|t_{n}-y_{n}\right\| \\
& \leq \lambda_{n} L\left\|u_{n}-y_{n}\right\|\left\|t_{n}-y_{n}\right\| .
\end{aligned}
$$

Hence it follows from (3.5) and (3.7) that

$$
\begin{aligned}
\left\|t_{n}-x^{*}\right\|^{2} & \leq\left\|u_{n}-x^{*}\right\|^{2}-\left\|u_{n}-y_{n}\right\|^{2}-\left\|y_{n}-t_{n}\right\|^{2}+2 \lambda_{n} L\left\|u_{n}-y_{n}\right\|\left\|t_{n}-y_{n}\right\| \\
& \leq\left\|u_{n}-x^{*}\right\|^{2}-\left\|u_{n}-y_{n}\right\|^{2}-\left\|y_{n}-t_{n}\right\|^{2}+\lambda_{n} L\left(\left\|u_{n}-y_{n}\right\|^{2}+\left\|y_{n}-t_{n}\right\|^{2}\right) \\
& =\left\|u_{n}-x^{*}\right\|^{2}+\left(\lambda_{n} L-1\right)\left\|u_{n}-y_{n}\right\|^{2}+\left(\lambda_{n} L-1\right)\left\|y_{n}-t_{n}\right\|^{2} .
\end{aligned}
$$


Since $\lambda_{n} \rightarrow 0$ as $n \rightarrow \infty$, there exists a positive integer $N_{0}$ such that $\lambda_{n} L-1 \leq-1 / 3$, when $n \geq N_{0}$. Hence it follows from (3.8) that

$$
\left\|t_{n}-x^{*}\right\| \leq\left\|u_{n}-x^{*}\right\|
$$

Observe that

$$
\left\|u_{n}-x^{*}\right\|=\left\|T_{r_{n}} x_{n}-T_{r_{n}} x^{*}\right\| \leq\left\|x_{n}-x^{*}\right\|
$$

and hence

$$
\left\|t_{n}-x^{*}\right\| \leq\left\|x_{n}-x^{*}\right\|
$$

Thus, we can calculate

$$
\begin{aligned}
\left\|x_{n+1}-x^{*}\right\| & =\left\|\alpha_{n} f\left(x_{n}\right)+\beta_{n} x_{n}+\gamma_{n} S_{n} t_{n}-x^{*}\right\| \\
& \leq \alpha_{n}\left\|f\left(x_{n}\right)-x^{*}\right\|+\beta_{n}\left\|x_{n}-x^{*}\right\|+\gamma_{n}\left\|t_{n}-x^{*}\right\| \\
& \leq \alpha_{n}\left\|f\left(x_{n}\right)-f\left(x^{*}\right)\right\|+\alpha_{n}\left\|f\left(x^{*}\right)-x^{*}\right\|+\beta_{n}\left\|x_{n}-x^{*}\right\|+\gamma_{n}\left\|x_{n}-x^{*}\right\| \\
& \leq\left(1-\alpha_{n}(1-\alpha)\right)\left\|x_{n}-x^{*}\right\|+\alpha_{n}\left\|f\left(x^{*}\right)-x^{*}\right\| \\
& =\left(1-\alpha_{n}(1-\alpha)\right)\left\|x_{n}-x^{*}\right\|+\alpha_{n}(1-\alpha) \frac{\left\|f\left(x^{*}\right)-x^{*}\right\|}{(1-\alpha)} .
\end{aligned}
$$

It follows from induction that

$$
\left\|x_{n}-x^{*}\right\| \leq \max \left\{\left\|x_{1}-x^{*}\right\|, \frac{\left\|f\left(x^{*}\right)-x^{*}\right\|}{1-\alpha}\right\}, \quad n \geq N_{0} .
$$

Therefore, $\left\{x_{n}\right\}$ is bounded. Hence, so are $\left\{t_{n}\right\},\left\{S_{n} t_{n}\right\},\left\{A u_{n}\right\},\left\{A y_{n}\right\}$, and $\left\{f\left(x_{n}\right)\right\}$.

Step $2\left(\lim _{n \rightarrow \infty}\left\|x_{n+1}-x_{n}\right\|=0\right)$. Indeed, we observe that for any $x, y \in C$,

$$
\begin{aligned}
\left\|\left(I-\lambda_{n} A\right) x-\left(I-\lambda_{n} A\right) y\right\|^{2} & =\left\|(x-y)-\lambda_{n}(A x-A y)\right\|^{2} \\
& =\|x-y\|^{2}-2 \lambda_{n}\langle x-y, A x-A y\rangle+\lambda_{n}^{2}\|A x-A y\|^{2} \\
& \leq\|x-y\|^{2}+\lambda_{n}^{2} L^{2}\|x-y\|^{2} \\
& =\left(1+\lambda_{n}^{2} L^{2}\right)\|x-y\|^{2},
\end{aligned}
$$

which implies that

$$
\left\|\left(I-\lambda_{n} A\right) x-\left(I-\lambda_{n} A\right) y\right\| \leq\left(1+\lambda_{n} L\right)\|x-y\| .
$$

Thus

$$
\begin{aligned}
\left\|t_{n+1}-t_{n}\right\| \leq & \left\|P_{C}\left(u_{n+1}-\lambda_{n+1} A y_{n+1}\right)-P_{C}\left(u_{n}-\lambda_{n} A y_{n}\right)\right\| \\
\leq & \left\|u_{n+1}-\lambda_{n+1} A y_{n+1}-\left(u_{n}-\lambda_{n} A y_{n}\right)\right\| \\
= & \left\|\left(u_{n+1}-\lambda_{n+1} A u_{n+1}\right)-\left(u_{n}-\lambda_{n+1} A u_{n}\right)+\lambda_{n+1}\left(A u_{n+1}-A y_{n+1}-A u_{n}\right)+\lambda_{n} A y_{n}\right\| \\
\leq & \left\|\left(u_{n+1}-\lambda_{n+1} A u_{n+1}\right)-\left(u_{n}-\lambda_{n+1} A u_{n}\right)\right\| \\
& +\lambda_{n+1}\left(\left\|A u_{n+1}\right\|+\left\|A y_{n+1}\right\|+\left\|A u_{n}\right\|\right)+\lambda_{n}\left\|A y_{n}\right\| \\
\leq & \left(1+\lambda_{n+1} L\right)\left\|u_{n+1}-u_{n}\right\|+\lambda_{n+1}\left(\left\|A u_{n+1}\right\|+\left\|A y_{n+1}\right\|+\left\|A u_{n}\right\|\right)+\lambda_{n}\left\|A y_{n}\right\| .
\end{aligned}
$$


On the other hand, from $u_{n}=T_{r_{n}} x_{n}$ and $u_{n+1}=T_{r_{n+1}} x_{n+1}$, we note that

$$
\begin{gathered}
\phi\left(u_{n}, y\right)+\frac{1}{r_{n}}\left\langle y-u_{n}, u_{n}-x_{n}\right\rangle \geq 0 \quad \forall y \in C, \\
\phi\left(u_{n+1}, y\right)+\frac{1}{r_{n+1}}\left\langle y-u_{n+1}, u_{n+1}-x_{n+1}\right\rangle \geq 0 \quad \forall y \in C .
\end{gathered}
$$

Putting $y=u_{n+1}$ in (3.17) and $y=u_{n}$ in (3.18), we have

$$
\begin{gathered}
\phi\left(u_{n}, u_{n+1}\right)+\frac{1}{r_{n}}\left\langle u_{n+1}-u_{n}, u_{n}-x_{n}\right\rangle \geq 0, \\
\phi\left(u_{n+1}, u_{n}\right)+\frac{1}{r_{n+1}}\left\langle u_{n}-u_{n+1}, u_{n+1}-x_{n+1}\right\rangle \geq 0 .
\end{gathered}
$$

So, from (A2), we have

$$
\left\langle u_{n+1}-u_{n}, \frac{u_{n}-x_{n}}{r_{n}}-\frac{u_{n+1}-x_{n+1}}{r_{n+1}}\right\rangle \geq 0
$$

and hence

$$
\left\langle u_{n+1}-u_{n}, u_{n}-u_{n+1}+u_{n+1}-x_{n}-\frac{r_{n}}{r_{n+1}}\left(u_{n+1}-x_{n+1}\right)\right\rangle \geq 0
$$

Without loss of generality, let us assume that there exists a real number $c$ such that $r_{n}>c>0$ for all $n \in \mathbb{N}$. Then, we have

$$
\begin{aligned}
\left\|u_{n+1}-u_{n}\right\|^{2} & \leq\left\langle u_{n+1}-u_{n}, x_{n+1}-x_{n}+\left(1-\frac{r_{n}}{r_{n+1}}\right)\left(u_{n+1}-x_{n+1}\right)\right\rangle \\
& \leq\left\|u_{n+1}-u_{n}\right\|\left\{\left\|x_{n+1}-x_{n}\right\|+\left|1-\frac{r_{n}}{r_{n+1}}\right|\left\|u_{n+1}-x_{n+1}\right\|\right\}
\end{aligned}
$$

and hence

$$
\begin{aligned}
\left\|u_{n+1}-u_{n}\right\| & \leq\left\|x_{n+1}-x_{n}\right\|+\frac{1}{r_{n+1}}\left|r_{n+1}-r_{n}\right|\left\|u_{n+1}-x_{n+1}\right\| \\
& \leq\left\|x_{n+1}-x_{n}\right\|+\frac{1}{c}\left|r_{n+1}-r_{n}\right| M
\end{aligned}
$$

where $M=\sup \left\{\left\|u_{n}-x_{n}\right\|: n \in \mathbb{N}\right\}$. It follows from (3.16) and the last inequality that

$$
\begin{aligned}
\left\|t_{n+1}-t_{n}\right\| \leq & \left(1+\lambda_{n+1} L\right)\left\|x_{n+1}-x_{n}\right\|+\left(1+\lambda_{n+1} L\right) \frac{1}{c}\left|r_{n+1}-r_{n}\right| M \\
& +\lambda_{n+1}\left(\left\|A u_{n+1}\right\|+\left\|A y_{n+1}\right\|+\left\|A u_{n}\right\|\right)+\lambda_{n}\left\|A y_{n}\right\| .
\end{aligned}
$$


Setting $z_{n}=\left(\alpha_{n} f\left(x_{n}\right)+\gamma_{n} S_{n} t_{n}\right) /\left(1-\beta_{n}\right)$, we obtain $x_{n+1}=\left(1-\beta_{n}\right) z_{n}+\beta_{n} x_{n}$ for all $n \in \mathbb{N}$. Thus, we have

$$
\begin{aligned}
\left\|z_{n+1}-z_{n}\right\|= & \left\|\frac{\alpha_{n+1} f\left(x_{n+1}\right)+\gamma_{n+1} S_{n+1} t_{n+1}}{1-\beta_{n+1}}-\frac{\alpha_{n} f\left(x_{n}\right)+\gamma_{n} S_{n} t_{n}}{1-\beta_{n}}\right\| \\
= & \| \frac{\alpha_{n+1}}{1-\beta_{n+1}} f\left(x_{n+1}\right)+\frac{\gamma_{n+1}}{1-\beta_{n+1}}\left(S_{n+1} t_{n+1}-S_{n} t_{n}\right)-\frac{\alpha_{n}}{1-\beta_{n}} f\left(x_{n}\right) \\
& -\left(1-\frac{\alpha_{n}}{1-\beta_{n}}\right) S_{n} t_{n}+\left(1-\frac{\alpha_{n+1}}{1-\beta_{n+1}} S_{n} t_{n}\right) \| \\
\leq & \frac{\alpha_{n+1}}{1-\beta_{n+1}}\left\|f\left(x_{n+1}\right)-S_{n} t_{n}\right\|+\frac{\alpha_{n}}{1-\beta_{n}}\left\|S_{n} t_{n}-f\left(x_{n}\right)\right\| \\
& +\frac{\gamma_{n+1}}{1-\beta_{n+1}}\left\|S_{n+1} t_{n+1}-S_{n} t_{n}\right\| .
\end{aligned}
$$

It follows from (3.24) that

$$
\begin{aligned}
\left\|S_{n+1} t_{n+1}-S_{n} t_{n}\right\| \leq & \left\|S_{n+1} t_{n+1}-S_{n+1} t_{n}\right\|+\left\|S_{n+1} t_{n}-S_{n} t_{n}\right\| \\
\leq & \left\|t_{n+1}-t_{n}\right\|+\left\|S_{n+1} t_{n}-S_{n} t_{n}\right\| \\
\leq & \left(1+\lambda_{n+1} L\right)\left\|x_{n+1}-x_{n}\right\|+\left(1+\lambda_{n+1} L\right) \frac{1}{c}\left|r_{n+1}-r_{n}\right| M \\
& +\lambda_{n+1}\left(\left\|A u_{n+1}\right\|+\left\|A y_{n+1}\right\|+\left\|A u_{n}\right\|\right)+\lambda_{n}\left\|A y_{n}\right\|+\left\|S_{n+1} t_{n}-S_{n} t_{n}\right\| .
\end{aligned}
$$

Combining (3.25) and (3.26), we have

$$
\begin{aligned}
\left\|z_{n+1}-z_{n}\right\|-\left\|x_{n+1}-x_{n}\right\| \leq & \frac{\alpha_{n+1}}{1-\beta_{n+1}}\left\|f\left(x_{n+1}\right)-S_{n} t_{n}\right\|+\frac{\alpha_{n}}{1-\beta_{n}}\left\|S_{n} t_{n}-f\left(x_{n}\right)\right\| \\
& +\frac{\gamma_{n+1}}{1-\beta_{n+1}}\left(1+\lambda_{n+1} L\right)\left\|x_{n+1}-x_{n}\right\|+\frac{\gamma_{n+1}}{1-\beta_{n+1}}\left(1+\lambda_{n+1} L\right) \frac{1}{c}\left|r_{n+1}-r_{n}\right| M \\
& +\frac{\gamma_{n+1}}{1-\beta_{n+1}} \lambda_{n+1}\left(\left\|A u_{n+1}\right\|+\left\|A y_{n+1}\right\|+\left\|A u_{n}\right\|\right)+\frac{\gamma_{n+1}}{1-\beta_{n+1}} \lambda_{n}\left\|A y_{n}\right\| \\
& +\frac{\gamma_{n+1}}{1-\beta_{n+1}}\left\|S_{n+1} t_{n}-S_{n} t_{n}\right\|-\left\|x_{n+1}-x_{n}\right\| \\
\leq & \frac{\alpha_{n+1}}{1-\beta_{n+1}}\left\|f\left(x_{n+1}\right)-S_{n} t_{n}\right\|+\frac{\alpha_{n}}{1-\beta_{n}}\left\|S_{n} t_{n}-f\left(x_{n}\right)\right\| \\
& +\frac{\gamma_{n+1}}{1-\beta_{n+1}} \lambda_{n+1} L\left\|x_{n+1}-x_{n}\right\|+\frac{\gamma_{n+1}}{1-\beta_{n+1}}\left(1+\lambda_{n+1} L\right) \frac{1}{c}\left|r_{n+1}-r_{n}\right| M \\
& +\frac{\gamma_{n+1}}{1-\beta_{n+1}} \lambda_{n+1}\left(\left\|A u_{n+1}\right\|+\left\|A y_{n+1}\right\|+\left\|A u_{n}\right\|\right) \\
& +\frac{\gamma_{n+1}}{1-\beta_{n+1}} \lambda_{n}\left\|A y_{n}\right\|+\frac{\gamma_{n+1}}{1-\beta_{n+1}} \sup \left\{\left\|S_{n+1} t-S_{n} t\right\|: t \in\left\{t_{n}\right\}\right\} .
\end{aligned}
$$


This together with (C1)-(C5) and $\lim _{n \rightarrow \infty} \sup \left\{\left\|S_{n+1} t-S_{n} t\right\|: t \in\left\{t_{n}\right\}\right\}=0$ implies that

$$
\limsup _{n \rightarrow \infty}\left(\left\|z_{n+1}-z_{n}\right\|-\left\|x_{n+1}-x_{n}\right\|\right) \leq 0
$$

Hence, by Lemma 2.2, we obtain $\left\|z_{n}-x_{n}\right\| \rightarrow 0$ as $n \rightarrow \infty$. It then follows that

$$
\lim _{n \rightarrow \infty}\left\|x_{n+1}-x_{n}\right\|=\lim _{n \rightarrow \infty}\left(1-\beta_{n}\right)\left\|z_{n}-x_{n}\right\|=0 .
$$

By (3.23) and (3.24), we also have

$$
\lim _{n \rightarrow \infty}\left\|t_{n+1}-t_{n}\right\|=\lim _{n \rightarrow \infty}\left\|u_{n+1}-u_{n}\right\|=0 .
$$

Step $3\left(\lim _{n \rightarrow \infty}\left\|S t_{n}-t_{n}\right\|=0\right)$. Indeed, pick any $x^{*} \in \cap_{n=1}^{\infty} F\left(S_{n}\right) \cap \operatorname{VI}(A, C) \cap \operatorname{EP}(\phi)$, to obtain

$$
\begin{aligned}
\left\|u_{n}-x^{*}\right\|^{2} & =\left\|T_{r_{n}} x_{n}-T_{r_{n}} x^{*}\right\|^{2} \leq\left\langle T_{r_{n}} x_{n}-T_{r_{n}} x^{*}, x_{n}-x^{*}\right\rangle=\left\langle u_{n}-x^{*}, x_{n}-x^{*}\right\rangle \\
& =\frac{1}{2}\left(\left\|u_{n}-x^{*}\right\|^{2}+\left\|x_{n}-x^{*}\right\|^{2}-\left\|x_{n}-u_{n}\right\|^{2}\right) .
\end{aligned}
$$

Therefore, $\left\|u_{n}-x^{*}\right\|^{2} \leq\left\|x_{n}-x^{*}\right\|^{2}-\left\|x_{n}-u_{n}\right\|^{2}$. From Lemma 2.1 and (3.9), we obtain, when $n \geq N_{0}$, that

$$
\begin{aligned}
\left\|x_{n+1}-x^{*}\right\|^{2} & =\left\|\alpha_{n} f\left(x_{n}\right)+\beta_{n} x_{n}+\gamma_{n} S_{n} t_{n}-x^{*}\right\|^{2} \\
& \leq \alpha_{n}\left\|f\left(x_{n}\right)-x^{*}\right\|^{2}+\beta_{n}\left\|x_{n}-x^{*}\right\|^{2}+\gamma_{n}\left\|S_{n} t_{n}-x^{*}\right\|^{2} \\
& \leq \alpha_{n}\left\|f\left(x_{n}\right)-x^{*}\right\|^{2}+\beta_{n}\left\|x_{n}-x^{*}\right\|^{2}+\gamma_{n}\left\|t_{n}-x^{*}\right\|^{2} \\
& \leq \alpha_{n}\left\|f\left(x_{n}\right)-x^{*}\right\|^{2}+\beta_{n}\left\|x_{n}-x^{*}\right\|^{2}+\gamma_{n}\left\|u_{n}-x^{*}\right\|^{2} \\
& \leq \alpha_{n}\left\|f\left(x_{n}\right)-x^{*}\right\|^{2}+\beta_{n}\left\|x_{n}-x^{*}\right\|^{2}+\gamma_{n}\left(\left\|x_{n}-x^{*}\right\|^{2}-\left\|x_{n}-u_{n}\right\|^{2}\right) \\
& \leq \alpha_{n}\left\|f\left(x_{n}\right)-x^{*}\right\|^{2}+\left(1-\alpha_{n}\right)\left\|x_{n}-x^{*}\right\|^{2}-\gamma_{n}\left\|x_{n}-u_{n}\right\|^{2}
\end{aligned}
$$

and hence

$$
\begin{aligned}
r_{n}\left\|x_{n}-u_{n}\right\|^{2} & \leq \alpha_{n}\left\|f\left(x_{n}\right)-x^{*}\right\|^{2}+\left\|x_{n}-x^{*}\right\|^{2}-\left\|x_{n+1}-x^{*}\right\|^{2} \\
& \leq \alpha_{n}\left\|f\left(x_{n}\right)-x^{*}\right\|^{2}+\left\|x_{n}-x_{n+1}\right\|\left(\left\|x_{n}-x^{*}\right\|+\left\|x_{n+1}-x^{*}\right\|\right) .
\end{aligned}
$$

It now follows from the last inequality, (C1), (C2), (C3) and (3.29), that

$$
\lim _{n \rightarrow \infty}\left\|x_{n}-u_{n}\right\|=0 .
$$

Noting that

$$
\begin{aligned}
\left\|y_{n}-x_{n}\right\| & =\left\|P_{C}\left(u_{n}-\lambda_{n} A u_{n}\right)-x_{n}\right\| \leq\left\|u_{n}-x_{n}\right\|+\lambda_{n}\left\|A u_{n}\right\| \longrightarrow 0 \quad \text { as } n \longrightarrow \infty \\
\left\|y_{n}-t_{n}\right\| & =\left\|P_{C}\left(u_{n}-\lambda_{n} A u_{n}\right)-P_{C}\left(u_{n}-\lambda_{n} A y_{n}\right)\right\| \leq \lambda_{n}\left\|A u_{n}-A y_{n}\right\| \longrightarrow 0 \text { as } n \longrightarrow \infty
\end{aligned}
$$


Thus

$$
\left\|t_{n}-x_{n}\right\| \leq\left\|t_{n}-y_{n}\right\|+\left\|y_{n}-x_{n}\right\| \longrightarrow 0 \quad \text { as } n \longrightarrow \infty \text {. }
$$

We note that

$$
\begin{aligned}
\left\|S_{n} y_{n}-x_{n+1}\right\| & \leq\left\|S_{n} y_{n}-S_{n} t_{n}\right\|+\left\|S_{n} t_{n}-x_{n+1}\right\| \\
& \leq\left\|y_{n}-t_{n}\right\|+\alpha_{n}\left\|S_{n} t_{n}-f\left(x_{n}\right)\right\|+\beta_{n}\left\|S_{n} t_{n}-x_{n}\right\| \\
& \leq\left\|y_{n}-t_{n}\right\|+\alpha_{n}\left\|S_{n} t_{n}-f\left(x_{n}\right)\right\|+\beta_{n}\left\|S_{n} t_{n}-S_{n} x_{n}\right\|+\beta_{n}\left\|S_{n} x_{n}-x_{n}\right\| \\
& \leq\left\|y_{n}-t_{n}\right\|+\alpha_{n}\left\|S_{n} t_{n}-f\left(x_{n}\right)\right\|+\beta_{n}\left\|t_{n}-x_{n}\right\|+\beta_{n}\left\|S_{n} x_{n}-x_{n}\right\| .
\end{aligned}
$$

Using (3.37), we have

$$
\begin{aligned}
\left\|S_{n} x_{n}-x_{n}\right\| \leq & \left\|S_{n} x_{n}-S_{n} y_{n}\right\|+\left\|S_{n} y_{n}-x_{n+1}\right\|+\left\|x_{n+1}-x_{n}\right\| \\
\leq & \left\|x_{n}-y_{n}\right\|+\left\|y_{n}-t_{n}\right\|+\alpha_{n}\left\|S_{n} t_{n}-f\left(x_{n}\right)\right\|+\beta_{n}\left\|t_{n}-x_{n}\right\| \\
& +\beta_{n}\left\|S_{n} x_{n}-x_{n}\right\|+\left\|x_{n+1}-x_{n}\right\|,
\end{aligned}
$$

so that

$$
\left(1-\beta_{n}\right)\left\|S_{n} x_{n}-x_{n}\right\| \leq\left\|x_{n}-y_{n}\right\|+\left\|y_{n}-t_{n}\right\|+\alpha_{n}\left\|S_{n} t_{n}-f\left(x_{n}\right)\right\|+\beta_{n}\left\|t_{n}-x_{n}\right\|+\left\|x_{n+1}-x_{n}\right\| .
$$

This implies that

$$
\lim _{n \rightarrow \infty}\left\|S_{n} x_{n}-x_{n}\right\|=0
$$

It now follows from (3.36) and (3.40) that

$$
\begin{aligned}
\left\|S_{n} t_{n}-t_{n}\right\| & \leq\left\|S_{n} t_{n}-S_{n} x_{n}\right\|+\left\|S_{n} x_{n}-x_{n}\right\|+\left\|x_{n}-t_{n}\right\| \\
& \leq 2\left\|t_{n}-x_{n}\right\|+\left\|S_{n} x_{n}-x_{n}\right\| \longrightarrow 0 \text { as } n \longrightarrow \infty .
\end{aligned}
$$

Applying Lemma 2.4 and (3.41), we have

$$
\begin{aligned}
\left\|S t_{n}-t_{n}\right\| & \leq\left\|S t_{n}-S_{n} t_{n}\right\|+\left\|S_{n} t_{n}-t_{n}\right\| \\
& \leq \sup \left\{\left\|S t-S_{n} t\right\|: t \in\left\{t_{n}\right\}\right\}+\left\|S_{n} t_{n}-t_{n}\right\| \longrightarrow 0 .
\end{aligned}
$$

It follows from the last inequality and (3.36) that

$$
\left\|x_{n}-S t_{n}\right\| \leq\left\|x_{n}-t_{n}\right\|+\left\|t_{n}-S t_{n}\right\| \longrightarrow 0 \quad \text { as } n \longrightarrow \infty .
$$

Step $4\left(\lim \sup _{n \rightarrow \infty}\left\langle f(q)-q, x_{n}-q\right\rangle \leq 0\right)$. Indeed, we choose a subsequence $\left\{t_{n_{i}}\right\}$ of $\left\{t_{n}\right\}$ such that

$$
\limsup _{n \rightarrow \infty}\left\langle f(q)-q, S t_{n}-q\right\rangle=\lim _{i \rightarrow \infty}\left\langle f(q)-q, S t_{n_{i}}-q\right\rangle
$$


Without loss of generality, we may assume that $\left\{t_{n_{i}}\right\}$ converges weakly to $z \in C$. From $\| S t_{n}-$ $t_{n} \| \rightarrow 0$, we obtain $S t_{n_{i}} \rightarrow z$. Now, we will show that $z \in F(S) \cap \operatorname{VI}(A, C) \cap \operatorname{EP}(\phi)$. Firstly, we will show $z \in \operatorname{EP}(\phi)$. Indeed, we observe that $u_{n}=T_{r_{n}} x_{n}$, and

$$
\phi\left(u_{n}, y\right)+\frac{1}{r_{n}}\left\langle y-u_{n}, u_{n}-x_{n}\right\rangle \geq 0 \quad \forall y \in C .
$$

From (A2), we also have

$$
\frac{1}{r_{n}}\left\langle y-u_{n}, u_{n}-x_{n}\right\rangle \geq \phi\left(y, u_{n}\right)
$$

and hence

$$
\left\langle y-u_{n_{i}}, \frac{u_{n_{i}}-x_{n_{i}}}{r_{n_{i}}}\right\rangle \geq \phi\left(y, u_{n_{i}}\right) .
$$

From $\left\|u_{n}-x_{n}\right\| \rightarrow 0,\left\|x_{n}-S t_{n}\right\| \rightarrow 0$, and $\left\|S t_{n}-t_{n}\right\| \rightarrow 0$, we get $u_{n_{i}} \rightarrow z$. Since $\left(u_{n_{i}}-x_{n_{i}}\right) / r_{n_{i}} \rightarrow$ 0 , it follows by (A4) that $0 \geq \phi(y, z)$ for all $y \in C$. For $t$ with $0<t \leq 1$ and $y \in C$, let $y_{t}=t y+(1-t) z$. Since $y \in C$ and $z \in C$, we have $y_{t} \in C$ and hence $\phi\left(y_{t}, z\right) \leq 0$. So, from (A1) and (A4), we have

$$
0=\phi\left(y_{t}, y_{t}\right) \leq t \phi\left(y_{t}, y\right)+(1-t) \phi\left(y_{t}, z\right) \leq t \phi\left(y_{t}, y\right)
$$

and hence $0 \leq \phi\left(y_{t}, y\right)$. From (A3), we have $0 \leq \phi(z, y)$ for all $y \in C$, and hence $z \in \operatorname{EP}(\phi)$. By the Opial's condition, we can obtain that $z \in F(S)$. Next we will show that $z \in \operatorname{VI}(A, C)$. Let

$$
T v= \begin{cases}A v+N_{C} v, & v \in C ; \\ \varnothing, & v \notin C .\end{cases}
$$

Then $T$ is maximal monotone (see [14]). Let $(v, w) \in G(T)$. Since $w-A v \in N_{C}(v)$ and $t_{n} \in C$, we have $\left\langle v-t_{n}, w-A v\right\rangle \geq 0$. On the other hand, from $t_{n}=P_{C}\left(u_{n}-\lambda_{n} A y_{n}\right)$, we have

$$
\left\langle v-t_{n}, t_{n}-\left(u_{n}-\lambda_{n} A y_{n}\right)\right\rangle \geq 0
$$

that is,

$$
\left\langle v-t_{n}, \frac{t_{n}-u_{n}}{\lambda_{n}}+A y_{n}\right\rangle \geq 0
$$

Therefore, we obtain

$$
\begin{aligned}
\left\langle v-t_{n_{i}}, w\right\rangle \geq\left\langle v-t_{n_{i}}, A v\right\rangle & \geq\left\langle v-t_{n_{i}}, A v\right\rangle-\left\langle v-t_{n_{i}}, \frac{t_{n_{i}}-u_{n_{i}}}{\lambda_{n_{i}}}+A y_{n_{i}}\right\rangle \\
& =\left\langle v-t_{n_{i}}, A v-A y_{n_{i}}-\frac{t_{n_{i}}-u_{n_{i}}}{\lambda_{n_{i}}}\right\rangle \\
& =\left\langle v-t_{n_{i}}, A v-A t_{n_{i}}\right\rangle+\left\langle v-t_{n_{i}}, A t_{n_{i}}-A y_{n_{i}}\right\rangle-\left\langle v-t_{n_{i}}, \frac{t_{n_{i}}-u_{n_{i}}}{\lambda_{n_{i}}}\right\rangle \\
& \geq\left\langle v-t_{n_{i}}, A t_{n_{i}}\right\rangle-\left\langle v-t_{n_{i}}, \frac{t_{n_{i}}-u_{n_{i}}}{\lambda_{n_{i}}}+A y_{n_{i}}\right\rangle \\
& =\left\langle v-t_{n_{i}}, A t_{n_{i}}-A y_{n_{i}}\right\rangle-\left\langle v-t_{n_{i}}, \frac{t_{n_{i}}-u_{n_{i}}}{\lambda_{n_{i}}}\right\rangle .
\end{aligned}
$$


Noting that $\left\|t_{n_{i}}-y_{n_{i}}\right\| \rightarrow 0,\left\|t_{n_{i}}-u_{n_{i}}\right\| \rightarrow 0$ as $n \rightarrow \infty, A$ is Lipschitz continuous and (3.52), we obtain

$$
\langle v-z, w\rangle \geq 0
$$

Since $T$ is maximal monotone, we have $z \in T^{-1} 0$, and hence $z \in \operatorname{VI}(A, C)$. Hence $z \in F(S) \cap$ $\mathrm{VI}(A, C) \cap \mathrm{EP}(\phi)$. The property of the metric projection implies that

$$
\begin{aligned}
\limsup _{n \rightarrow \infty}\left\langle f(q)-q, x_{n}-q\right\rangle & =\limsup _{n \rightarrow \infty}\left\langle f(q)-q, S t_{n}-q\right\rangle \\
& =\lim _{i \rightarrow \infty}\left\langle f(q)-q, S t_{n_{i}}-q\right\rangle=\langle f(q)-q, z-q\rangle \leq 0 .
\end{aligned}
$$

Step $5\left(\lim _{n \rightarrow \infty}\left\|x_{n}-q\right\|=0\right)$. Indeed, we observe that

$$
\begin{aligned}
\left\|x_{n+1}-q\right\|^{2}= & \left\langle\alpha_{n} f\left(x_{n}\right)+\beta_{n} x_{n}+\gamma_{n} S_{n} t_{n}, x_{n+1}-q\right\rangle \\
= & \alpha_{n}\left\langle f\left(x_{n}\right)-q, x_{n+1}-q\right\rangle+\beta_{n}\left\langle x_{n}-q, x_{n+1}-q\right\rangle+\gamma_{n}\left\langle S_{n} t_{n}-q, x_{n+1}-q\right\rangle \\
\leq & \frac{1}{2} \beta_{n}\left(\left\|x_{n}-q\right\|^{2}+\left\|x_{n+1}-q\right\|^{2}\right)+\frac{1}{2} \gamma_{n}\left(\left\|t_{n}-q\right\|^{2}+\left\|x_{n+1}-q\right\|^{2}\right) \\
& +\alpha_{n}\left\langle f\left(x_{n}\right)-f(q), x_{n+1}-q\right\rangle+\alpha_{n}\left\langle f(q)-q, x_{n+1}-q\right\rangle \\
\leq & \frac{1}{2}\left(1-\alpha_{n}\right)\left(\left\|x_{n}-q\right\|^{2}+\left\|x_{n+1}-q\right\|^{2}\right)+\frac{1}{2} \alpha_{n}\left(\left\|f\left(x_{n}\right)-f(q)\right\|^{2}+\left\|x_{n+1}-q\right\|^{2}\right) \\
& +\alpha_{n}\left\langle f(q)-q, x_{n+1}-q\right\rangle \\
\leq & \frac{1}{2}\left(1-\alpha_{n}\left(1-\alpha^{2}\right)\right)\left\|x_{n}-q\right\|^{2}+\frac{1}{2}\left(1-\alpha_{n}\right)\left\|x_{n+1}-q\right\|^{2} \\
& +\frac{1}{2} \alpha_{n}\left\|x_{n+1}-q\right\|^{2}+\alpha_{n}\left\langle f(q)-q, x_{n+1}-q\right\rangle,
\end{aligned}
$$

which implies that

$$
\left\|x_{n+1}-q\right\|^{2} \leq\left(1-\alpha_{n}\left(1-\alpha^{2}\right)\right)\left\|x_{n}-q\right\|^{2}+2 \alpha_{n}\left\langle f(q)-q, x_{n+1}-q\right\rangle .
$$

Setting $\delta_{n}=2 \alpha_{n}\left\langle f(q)-q, x_{n+1}-q\right\rangle$, we have $\limsup _{n \rightarrow \infty}\left(\delta_{n} / \alpha_{n}\left(1-\alpha^{2}\right)\right) \leq 0$. Applying Lemma 2.3 to (3.56), we conclude that $\left\{x_{n}\right\}$ converges strongly to $q$. Consequently, $\left\{u_{n}\right\}$ and $\left\{y_{n}\right\}$ converge strongly to $q$. This completes the proof.

As in [12, Theorem 4.1], we can generate a sequence $\left\{S_{n}\right\}$ of nonexpansive mappings satisfying condition $\sum_{n=1}^{\infty} \sup \left\{\left\|S_{n+1} z-S_{n} z\right\|: z \in B\right\}<\infty$ for any bounded subset $B$ of $C$ by using convex combination of a general sequence $\left\{T_{k}\right\}$ of nonexpansive mappings with a common fixed point.

Corollary 3.2. Let $C$ be a closed convex subset of a real Hilbert space $H$. Let $\phi$ be a bifunction from $C \times C$ to $\mathbb{R}$ satisfying (A1)-(A4), $A: C \rightarrow H$ a monotone, L-Lipschitz continuous mapping and let 
$\left\{\beta_{n}^{k}\right\}$ be a family of nonnegative numbers with indices $n, k \in N$ with $k \leq n$ such that

(i) $\sum_{k=1}^{n} \beta_{n}^{k}=1$ for all $n \in \mathbb{N}$;

(ii) $\lim _{n \rightarrow \infty} \beta_{n}^{k}>0$ for every $k \in \mathbb{N}$;

(iii) $\sum_{n=1}^{\infty} \sum_{k=1}^{n}\left|\beta_{n+1}^{k}-\beta_{n}^{k}\right|<\infty$.

Let $\left\{T_{k}\right\}$ be a sequence of nonexpansive mappings of $C$ into itself with $\cap_{k=1}^{\infty} F\left(T_{k}\right) \cap \operatorname{VI}(A, C) \cap$ $\mathrm{EP}(\phi) \neq \varnothing$. Let $x_{1}=x \in C$ and $\left\{x_{n}\right\},\left\{y_{n}\right\}$ and $\left\{u_{n}\right\}$ be the sequences generated by

$$
\begin{gathered}
x_{1}=x \in C \text { chosen arbitrary, } \\
\phi\left(u_{n}, y\right)+\frac{1}{r_{n}}\left\langle y-u_{n}, u_{n}-x_{n}\right\rangle \geq 0 \quad \forall y \in C, \\
y_{n}=P_{C}\left(u_{n}-\lambda_{n} A u_{n}\right), \\
x_{n+1}=\alpha_{n} f\left(x_{n}\right)+\beta_{n} x_{n}+\gamma_{n} \sum_{k=1}^{n} \beta_{n}^{k} T_{k} P_{C}\left(u_{n}-\lambda_{n} A y_{n}\right) \quad \forall n \geq 1,
\end{gathered}
$$

where $\left\{\alpha_{n}\right\},\left\{\beta_{n}\right\},\left\{\gamma_{n}\right\} \subseteq[0,1],\left\{\lambda_{n}\right\} \subseteq(0,1)$, and $\left\{r_{n}\right\} \subseteq(0, \infty)$ satisfy the following conditions:

(C1) $\alpha_{n}+\beta_{n}+\gamma_{n}=1$,

(C2) $\lim _{n \rightarrow \infty} \alpha_{n}=0, \sum_{n=1}^{\infty} \alpha_{n}=\infty$,

(C3) $0<\liminf _{n \rightarrow \infty} \beta_{n} \leq \lim \sup _{n \rightarrow \infty} \beta_{n}<1$,

(C4) $\lim _{n \rightarrow \infty} \lambda_{n}=0$,

(C5) $\liminf _{n \rightarrow \infty} r_{n}>0, \sum_{n=1}^{\infty}\left|r_{n+1}-r_{n}\right|<\infty$.

Then the sequences $\left\{x_{n}\right\},\left\{u_{n}\right\}$, and $\left\{y_{n}\right\}$ converge strongly to the same point $q \in \cap_{k=1}^{\infty} F\left(T_{k}\right) \cap$ $\mathrm{VI}(A, C) \cap \operatorname{EP}(\phi)$, where $q=P_{\cap_{k=1}^{\infty}} F\left(T_{k}\right) \cap \mathrm{VI}(A, C) \cap \mathrm{EP}(\phi) f(q)$.

Setting $S_{n} \equiv S$ and $f:=u$ in Theorem 3.1, we have the following result.

Corollary 3.3 (see [10, Theorem 3.1]). Let $C$ be a closed convex subset of a real Hilbert space H. Let $A: C \rightarrow H$ be a monotone, $L$-Lipschitz continuous mapping, and let $S$ be a nonexpansive mapping of $C$ into itself such that $F(S) \cap \operatorname{VI}(A, C) \neq \varnothing$. Suppose $x_{1}=u \in C$ and $\left\{x_{n}\right\},\left\{y_{n}\right\}$ are given by

$$
\begin{aligned}
y_{n} & =P_{C}\left(x_{n}-\lambda_{n} A x_{n}\right), \\
x_{n+1} & =\alpha_{n} u+\beta_{n} x_{n}+\gamma_{n} S P_{C}\left(x_{n}-\lambda_{n} A y_{n}\right),
\end{aligned}
$$

where $\left\{\lambda_{n}\right\},\left\{\alpha_{n}\right\},\left\{\beta_{n}\right\},\left\{\gamma_{n}\right\}$ are sequences in $[0,1]$ satisfying the following conditions:

(i) $\alpha_{n}+\beta_{n}+\gamma_{n}=1$,

(ii) $\lim _{n \rightarrow \infty} \alpha_{n}=0, \sum_{n=1}^{\infty} \alpha_{n}=\infty$,

(iii) $0<\liminf _{n \rightarrow \infty} \beta_{n} \leq \lim \sup _{n \rightarrow \infty} \beta_{n}<1$,

(iv) $\lim _{n \rightarrow \infty} \lambda_{n}=0$.

Then $\left\{x_{n}\right\}$ converges strongly to $P_{F(S) \cap V I(A, C)} u$.

Proof. Put $\phi(x, y)=0$ for all $x, y \in C$ and $\left\{r_{n}\right\}=1$ in Theorem 3.1. Thus, we have $u_{n}=x_{n}$. Then the sequence $\left\{x_{n}\right\}$ generated in Corallary 3.3 converges strongly to $P_{F(S) \cap V I(A, C)} u$. 


\section{Applications}

In this section, we consider the problem of finding a zero of a monotone operator. A multivalued operator $S: H \rightarrow 2^{H}$ with domain $D(S)=\{z \in H: S z \neq \varnothing\}$ and range $R(S)=\{S z: z \in D(T)\}$ is said to be monotone if for each $x_{i} \in D(S)$ and $y_{i} \in S x_{i}, i=1,2$, we have $\left\langle x_{1}-x_{2}, y_{1}-y_{2}\right\rangle \geq 0$. A monotone operator $S$ is said to be maximal if its graph $G(S)=\{(x, y): y \in S x\}$ is not properly contained in the graph of any other monotone operator. Let $I$ denote the identity operator on $H$ and let $S: H \rightarrow 2^{H}$ be a maximal monotone operator. Then we can define, for each $r>0$, a nonexpansive single-valued mapping $J_{r}: H \rightarrow H$ by $J_{r}=(I+r S)^{-1}$. It is called the resolvent (or the proximal mapping) of $S$. We also define the Yosida approximation $A_{r}$ by $A_{r}=\left(I-J_{r}\right) / r$. We know that $A_{r} x \in S J_{r} x$ and $\left\|A_{r} x\right\| \leq \inf \{\|y\|: y \in S x\}$ for all $x \in H$. We also know that $S^{-1} 0=F\left(J_{r}\right)$ for all $r>0$; see, for instance, Rockafellar [19] or Takahashi [20].

Lemma 4.1 (the resolvent identity). For $\lambda, \mu>0$, there holds the identity

$$
J_{\lambda} x=J_{\mu}\left(\frac{\mu}{\lambda}+\left(1-\frac{\mu}{\lambda}\right) J_{\lambda} x\right), \quad x \in H .
$$

By using Theorem 3.1 and Lemma 4.1, we may obtain the following improvement.

Theorem 4.2. Let $S: H \rightarrow 2^{H}$ be a maximal monotone operator. Let $\phi$ be a bifunction from $C \times C$ to $\mathbb{R}$ satisfying (A1)-(A4), $A: C \rightarrow H$ a monotone L-Lipschitz continuous mapping of $C$ into $H$ such that $S^{-1}(0) \cap \operatorname{VI}(A, C) \cap \mathrm{EP}(\phi) \neq \varnothing$ and $f$ a contraction of $C$ into itself with coefficient $\alpha \in(0,1)$. Let the sequences $\left\{x_{n}\right\},\left\{u_{n}\right\}$ and $\left\{y_{n}\right\}$ be generated by

$$
\begin{gathered}
x_{1}=x \in C \text { chosen arbitrary, } \\
\phi\left(u_{n}, y\right)+\frac{1}{r_{n}}\left\langle y-u_{n}, u_{n}-x_{n}\right\rangle \geq 0 \quad \forall y \in C, \\
y_{n}=P_{C}\left(u_{n}-\lambda_{n} A u_{n}\right), \\
x_{n+1}=\alpha_{n} f\left(x_{n}\right)+\beta_{n} x_{n}+\gamma_{n} J_{r_{n}} P_{C}\left(u_{n}-\lambda_{n} A y_{n}\right) \quad \forall n \geq 1,
\end{gathered}
$$

where $\left\{\alpha_{n}\right\},\left\{\beta_{n}\right\},\left\{\gamma_{n}\right\} \subseteq[0,1],\left\{\lambda_{n}\right\} \subseteq(0,1)$, and $\left\{r_{n}\right\} \subseteq(0, \infty)$ satisfy the following conditions:

(C1) $\alpha_{n}+\beta_{n}+\gamma_{n}=1$,

(C2) $\lim _{n \rightarrow \infty} \alpha_{n}=0, \sum_{n=1}^{\infty} \alpha_{n}=\infty$,

(C3) $0<\liminf _{n \rightarrow \infty} \beta_{n} \leq \lim \sup _{n \rightarrow \infty} \beta_{n}<1$,

(C4) $\lim _{n \rightarrow \infty} \lambda_{n}=0$,

(C5) $\liminf _{n \rightarrow \infty} r_{n}>0, \sum_{n=1}^{\infty}\left|r_{n+1}-r_{n}\right|<\infty$.

Then $\left\{x_{n}\right\}$ converges strongly to $q=P_{S^{-1}(0) \cap V I(A, C) \cap E P(\phi)} f(q)$.

Proof. We first verify that $\sum_{n=1}^{\infty} \sup \left\{\left\|J_{r_{n+1}} z-J_{r_{n}} z\right\|: z \in B\right\}<\infty$ for any bounded subset $B$ of $C$. Let $B$ be a bounded subset of $C$. Since $S^{-1}(0)=F\left(J_{r_{n}}\right)$ for each $n \in \mathbb{N},\left\{J_{r_{n}} z: z \in B, n \in \mathbb{N}\right\}$ is bounded. It follows from Lemma 4.1 that

$$
J_{r_{n+1}} z=J_{r_{n}}\left(\frac{r_{n}}{r_{n+1}} z+\left(1-\frac{r_{n}}{r_{n+1}}\right) J_{r_{n+1}} z\right), \quad z \in H
$$


Thus

$$
\begin{aligned}
\left\|J_{r_{n+1}} z-J_{r_{n}} z\right\| & \leq \frac{\left|r_{n+1}-r_{n}\right|}{r_{n+1}}\left\|J_{r_{n+1}} z-z\right\| \\
& \leq M\left|r_{n+1}-r_{n}\right|
\end{aligned}
$$

for each $z \in B$ and $n \in \mathbb{N}$, where $M=\sup \left\{\left\|J_{r_{n+1}} z-z\right\|: z \in B, n \in \mathbb{N}\right\} / \inf \left\{r_{n}: n \in \mathbb{N}\right\}$. Hence we get

$$
\sum_{n=1}^{\infty} \sup \left\{\left\|J_{r_{n+1}} z-J_{r_{n}} z\right\|: z \in B\right\} \leq M \sum_{n=1}^{\infty}\left|r_{n+1}-r_{n}\right|<\infty
$$

By the assumption that $\sum_{n=1}^{\infty}\left|r_{n+1}-r_{n}\right|<\infty$, we obtain $r_{n} \rightarrow r$ for some $r>0$. Since $\left\|J_{r} z-J_{r_{n}} z\right\| \leq$ $\left(\left|r-r_{n}\right| / r\right)\left\|z-J_{r} z\right\|$, we obtain that $\lim _{n \rightarrow \infty} J_{r_{n}} z=J_{r} z$ for all $z \in C$. Since $F\left(J_{\mu}\right)=S^{-1}(0)$ for all $\mu>0$, we have $F\left(J_{r}\right)=\cap_{n=1}^{\infty} F\left(J_{r_{n}}\right)=S^{-1}(0) \neq \varnothing$. Therefore, by Theorem 3.1, $\left\{x_{n}\right\}$ converges

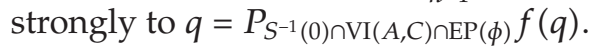

\section{Acknowledgments}

The author would like to thank the referees for reading this paper carefully, providing valuable suggestions and comments, and pointing out a major error in the original version of this paper. This research was partially supported by the Commission on Higher Education.

\section{References}

[1] S. D. Flåm and A. S. Antipin, "Equilibrium programming using proximal-like algorithms," Mathematical Programming, vol. 78, no. 1, pp. 29-41, 1997.

[2] J.-C. Yao and O. Chadli, "Pseudomonotone complementarity problems and variational inequalities," in Handbook of Generalized Convexity and Generalized Monotonicity, N. Hadjisavvas, S. Komlósi, and S. Schaible, Eds., vol. 76 of Nonconvex Optimization and Its Applications, pp. 501-558, Springer, New York, NY, USA, 2005.

[3] L. C. Zeng, S. Schaible, and J. C. Yao, "Iterative algorithm for generalized set-valued strongly nonlinear mixed variational-like inequalities," Journal of Optimization Theory and Applications, vol. 124, no. 3, pp. 725-738, 2005.

[4] F. E. Browder and W. V. Petryshyn, "Construction of fixed points of nonlinear mappings in Hilbert space," Journal of Mathematical Analysis and Applications, vol. 20, no. 2, pp. 197-228, 1967.

[5] F. Liu and M. Z. Nashed, "Regularization of nonlinear ill-posed variational inequalities and convergence rates," Set-Valued Analysis, vol. 6, no. 4, pp. 313-344, 1998.

[6] W. Takahashi and M. Toyoda, "Weak convergence theorems for nonexpansive mappings and monotone mappings," Journal of Optimization Theory and Applications, vol. 118, no. 2, pp. 417-428, 2003.

[7] G. M. Korpelevič, "An extragradient method for finding saddle points and for other problems," Èkonomika i Matematicheskie Metody, vol. 12, no. 4, pp. 747-756, 1976.

[8] N. Nadezhkina and W. Takahashi, "Weak convergence theorem by an extragradient method for nonexpansive mappings and monotone mappings," Journal of Optimization Theory and Applications, vol. 128, no. 1, pp. 191-201, 2006.

[9] L.-C. Zeng and J.-C. Yao, "Strong convergence theorem by an extragradient method for fixed point problems and variational inequality problems," Taiwanese Journal of Mathematics, vol. 10, no. 5, pp. 1293-1303, 2006.

[10] Y. Yao, Y.-C. Liou, and J.-C. Yao, "An extragradient method for fixed point problems and variational inequality problems," Journal of Inequalities and Applications, vol. 2007, Article ID 38752, 12 pages, 2007.

[11] S. Takahashi and W. Takahashi, "Viscosity approximation methods for equilibrium problems and fixed point problems in Hilbert spaces," Journal of Mathematical Analysis and Applications, vol. 331, no. 1, pp. 506-515, 2007. 
[12] K. Aoyama, Y. Kimura, W. Takahashi, and M. Toyoda, "Approximation of common fixed points of a countable family of nonexpansive mappings in a Banach space," Nonlinear Analysis: Theory, Methods Eamp; Applications, vol. 67, no. 8, pp. 2350-2360, 2007.

[13] W. Takahashi, Nonlinear Functional Analysis, Yokohama Publishers, Yokohama, Japan, 2000.

[14] R. T. Rockafellar, "On the maximality of sums of nonlinear monotone operators," Transactions of the American Mathematical Society, vol. 149, no. 1, pp. 75-88, 1970.

[15] M. O. Osilike and D. I. Igbokwe, "Weak and strong convergence theorems for fixed points of pseudocontractions and solutions of monotone type operator equations," Computers Eamp; Mathematics with Applications, vol. 40, no. 4-5, pp. 559-567, 2000.

[16] T. Suzuki, "Strong convergence of Krasnoselskii and Mann's type sequences for one-parameter nonexpansive semigroups without Bochner integrals," Journal of Mathematical Analysis and Applications, vol. 305, no. 1, pp. 227-239, 2005.

[17] H.-K. Xu, "Viscosity approximation methods for nonexpansive mappings," Journal of Mathematical Analysis and Applications, vol. 298, no. 1, pp. 279-291, 2004.

[18] E. Blum and W. Oettli, "From optimization and variational inequalities to equilibrium problems," The Mathematics Student, vol. 63, no. 1-4, pp. 123-145, 1994.

[19] R. T. Rockafellar, "Monotone operators and the proximal point algorithm," SIAM Journal on Control and Optimization, vol. 14, no. 5, pp. 877-898, 1976.

[20] W. Takahashi, Nonlinear Functional Analysis, Kindai Kagaku sha, Tokyo, Japan, 1988. 